\title{
A Clinical Study of Empyema Thoracis in Children Admitted in Tertiary Care Hospital
}

\author{
Authors \\ Dr Trinadh Naidu Chandaka ${ }^{1}$, Dr Jagadeeswararao Metta $^{2}$, Dr Attada Ajay Kumar ${ }^{3}$ \\ Dr Chinnarao $\mathbf{G}^{4}$ \\ ${ }^{1.2 .3}$ Postgraduate, Department of Pediatrics, Andhra Medical College \\ ${ }^{4}$ Assistant Professor, Department of Pediatrics, Andhra Medical College
}

\begin{abstract}
Introduction: Empyema thoracis, accumulation of pus in the pleural cavity, is a dreaded complication of pneumonia, is in increasing trend. Early antibiotics with intercoastal tube drainage, advanced interventions like intraplueral fibrinolytic therapy, VATS have greatly reduced the mortality and morbidity.

Aims and Objectives

To study clinical presentation, course, bacteriological, radiological features, management and outcome of empyema thoracis.

Methodology

Study Sample: 35 cases were studied during March 2017 to July 2018.

Inclusion Criteria: Children with pleural fluid with any of the characteristics like grossly purulent, loculation on ultrasonography, gram stain positive, WBC count $>10000 \mathrm{~mm} 3$ with neutrophilic predominance.

Exclusion Criteria: Secondary to trauma, surgery, tuberculosis.

Methods: Physical examination, Blood (CBC,ESR, Blood culture), Radiological (Xray, Usg, CECT), Thorococentesis (pleural fluid analysis).

Observations and Results: Male (60\%) >female(40\%), maximum incidence (45.7\%) was noted in 3-5 years age group with lowest incidence in below lyr(5.7\%). Major organism isolated was Staphylococcus aureus(17.14\%), Pneumococcal(11.42\%), pseudomonas(8.57\%), culture negative (51.42\%). Chest Xray with associated lung lesions like consolidation (82.85\%),collapse(45.71\%),mediastinal shift(34.28\%). 32 of 35 cases were treated with combination of IV antibiotics and chest tube drainage alone. 3cases underwent Decortication probably due to late presentation to hospital.

Conclusion: More common among males and in 3-5 age groups. More common etiological agent is Staphylococcus aureus. Mainstay of treatment is antibiotics with intercoastal tube drainage. Decortication done for loculated empyema where ICTD is inadequate. Early diagnosis, prompt treatment with antibiotics and ICTD, abates need for surgical interventions, decreases mortality and morbidity.
\end{abstract}

\section{Introduction}

Paediatric Pulmonology is a fascinating developing sub-specialty in the $21^{\text {st }}$ century. The respiratory illness carries an enormous magnitude of morbidity and mortality. Empyema thoracis, accumulation of pus in the pleural cavity or suppuration in the 
pleural cavity, has been recognised to be a serious problem for centuries. Empyema was a dreaded complication of pneumonia and chest injuries prior to antibiotic era.

Inspite of the advent of newer potent antibiotics, bacterial pneumonia and empyema and its complications still result in significant morbidity and mortality in children and this partly may be attributed to the prevalence of poverty, ignorance, illiteracy and poor compliance with treatment.

The evolution of modern non-invasive diagnostic technology made possible the recognition of the illness and clear understanding of specialized therapy like respiratory support, oxygen therapy, physiotherapy and nutritional support and specialized care with early intervention with advanced techniques like intrapleural fibrinolytic therapy, VATS go a long way to bring down the morbidity and mortality.

\section{Aims and Objectives}

- To study the epidemiological aspects of the disease.

- To study clinical presentation and course of empyema.

- To know the bacteriological agents in etiology of empyema.

- To study the radiological findings in empyema

- To study the effect of comorbidities (malnutrition and HIV) on outcome of empyema thoracis.

- To study the management, outcome, morbidity and mortality patterns of empyema.

\section{Materials and Methods}

35 cases of empyema were studied among total number of PICU admissions (<12years) at King George Hospital, Visakhapatnam during a period of 15 months from March 2017 to July 2018.

Inclusion criteria - children with pleural aspirate having any of the characteristics like - grossly purulent fluid, Gram stain positive, WBC count
$>10000$ with neutrophil predominance, loculated effusion on ultrasound.

Exclusion criteria: empyema secondary to trauma, surgery, tuberculous empyema.

\section{Methodology}

- History taking

- Physical examination

- Blood investigations - complete blood counts, ESR, HIV, blood culture

- Urine - albumin, sugar, microscopy

- Mantoux test

- Radiological - Xray chest (PA, lateral views), ultra sonogram chest HRCT chest, CECT chest wherever necessary

Thoracocentesis done in all cases and pleural fluid obtained was subjected to biochemical analysis, microbiological and pathological evaluations.

All cases were treated with antibiotics and intercostal tube drainage. In selected cases HRCT done and reffered for surgery. $\square$ All cases were followed during the course of hospital stay for response to therapy.

Presence of pleural fluid is determined by the physical examination, chest X-ray and ultrasound examinations. The area of maximum accumulation is judged from PA and lateral chest Xrays. It is confirmed by ultrasound examination. If the fluid is small, or if the fluid is loculated, ultrasonography is the method of choice to guide the thoracentesis.

\section{Thoracocentesis}

Position of the patient - Sitting upright, either resting backward over backrest or bending forward and resting head over a stool with a pillow. After sufficiently preparing the area and anaesthetizing, $22 \mathrm{G}$ or $24 \mathrm{G}$ needle is introduced in the intercostal space just above the upper border of lower rib and fluid removed for diagnostic purposes

Obtained pleural fluid is analyzed biochemically, pathologically and microbiologically to know the etiological factors and to manage the case appropriately 
Observations and Results

Occurrence of Empyema among PICU Admissions

Of the 5725 cases admitted in PICU, Department of pediatrics, King George Hospital, Visakhapatnam during the study period, 35 cases were having empyema which constitutes $0.6 \%$. Out of 35 cases of empyema 14 children were females comprising of $40 \%$ and 21 children were males comprising of $60 \%$. The incidence of empyema is more in male children as compared to female children.

Age and sex wise Distribution of Empyema Cases

\begin{tabular}{|c|c|c|c|c|}
\hline $\begin{array}{c}\text { Age groups } \\
\text { (in years) }\end{array}$ & Males & Females & Total & Percentage \\
\hline$<1$ & 1 & 1 & 2 & 5.71 \\
\hline $1-2$ & 6 & 4 & 10 & 28.57 \\
\hline $3-5$ & 10 & 6 & 16 & 45.71 \\
\hline $6-12$ & 4 & 3 & 7 & 20 \\
\hline Total & 21 & 14 & 35 & 100 \\
\hline
\end{tabular}

The above table showed, 2 cases were admitted in less than 1 year of age comprising of $5.71 \%, 10,16$ and 7 cases were admitted in between 1-2 years group, 3-5 years group and 6-12 years group respectively.

As per the above analysis, the incidence of empyema is less below the age of one year and as the age advances the incidence of the cases gradually increased and maximum incidence of cases $(45.71 \%)$ is noted in $3-5 \mathrm{yr}$ age group

\section{Empyema in relation to Malnutrition}

In present study empyema in relation to nutrition,11 cases were of normal weight comprising of $31.42 \%$. Remaining 24 cases were associated with malnutrition comprising of $68.57 \%$. Out of 35 cases , 2 cases $(5.71 \%)$ were HIV infected, of which 1 is a male and the other is female.

\section{Analysis of Symptomatology}

\begin{tabular}{|l|c|c|}
\hline Symptom & No of cases & Percentage \\
\hline Fever & 35 & 100 \\
\hline Cough & 31 & 88.57 \\
\hline Breathlessness & 35 & 100 \\
\hline Chest pain & 15 & 42.85 \\
\hline
\end{tabular}

In our study fever, breathlessness were present in all cases of empyema. Other symptoms like cough present in $88.57 \%$, chest pain was present in 15 cases comprising of $42.85 \%$.

\section{Duration of Illness}

In our study, majority of cases $(45.71 \%)$ presented to hospital or referred from initial therapy centre between 4 to 7 days of onset of symptoms. $8.57 \%$ ( 3 cases) of cases presented late (15 days).

Intercostal tube drainage was done within 8 to 15 days of onset of symptoms in most of the cases $(68.57 \%)$. It was delayed for more than 15 days of onset of illness in $14.28 \%$ (5 cases) of cases.

\section{Side of Accumulation of Empyema}

Out of 35 cases, 20 were having empyema on the right side comprising of $57.14 \%, 14$ cases were having empyema on the left side comprising of $40 \%$ and the remaining 1 case $(2.85 \%)$ had empyema bilaterally.

\section{Etiological Agents Causing Empyema}

\begin{tabular}{|l|c|c|c|c|}
\hline $\begin{array}{l}\text { MICROORGAN } \\
\text { ISM }\end{array}$ & $\mathbf{3} \mathbf{~ y r}$ & $\begin{array}{c}\mathbf{3 - 5} \\
\mathbf{y r}\end{array}$ & $\begin{array}{c}\mathbf{6}-\mathbf{1 2} \\
\mathbf{y r}\end{array}$ & TOTAL \\
\hline $\begin{array}{l}\text { Staphylococcus } \\
\text { aureus }\end{array}$ & 3 & 2 & 1 & $6(17.14 \%)$ \\
\hline $\begin{array}{l}\text { Streptococcus } \\
\text { pneumoniae }\end{array}$ & 0 & 4 & 0 & $4(11.42 \%)$ \\
\hline $\begin{array}{l}\text { Streptococcus } \\
\text { pyogenes }\end{array}$ & 0 & 1 & 0 & $1(2.85 \%)$ \\
\hline Pseudomonas & 2 & 1 & 0 & $3(8.57 \%)$ \\
\hline Klebsiella & 1 & 1 & 0 & $2(5.71 \%)$ \\
\hline Escherichia coli & 1 & 0 & 0 & $1(2.85 \%)$ \\
\hline Culture sterile & 5 & 7 & 6 & $18(51.42 \%)$ \\
\hline
\end{tabular}

Among the culture positive cases, staphylococcus aureus was the commonest organism comprising of $6(17.14 \%)$ cases, most were from $<3$ yr children. It was found to be the causative organism in HIV infected case from our study.

Pneumococci and Pseudomonas are the next common organisms comprising of 4 cases $(11.42 \%)$ and 3 cases $(8.57 \%)$ respectively. Klebsiella caused 2 cases $(2.85 \%)$ and Betahaemolytic streptococci caused $1(5.71 \%)$ case and E.Coli caused 1 case $(5.71 \%)$ of emypema. 
E.coli was isolated from patient of <3yrs age group, Streptococcus and Pneumococcus from 3-5 yrs age group, Pseudomonas and Klebsiella from below 5yrs age group.

Culture was positive in $58.3 \%$ children of age $<3$ yrs, $56.25 \%$ of $3-5 \mathrm{yr}$ children, but only $14.28 \%$ of $6-12 \mathrm{yr}$ children.

Pleural fluid analysis was in favour of exudate in all the cases. On cytological examination polymorph predominance was seen in all the cases.

\section{Culture and Antibiotic Sensitivity}

\begin{tabular}{|l|c|}
\hline ORGANISM & SENSITIVE ANTIBIOTICS \\
\hline $\begin{array}{l}\text { Staphylococcus } \\
\text { aureus }\end{array}$ & Cloxacillin, Amoxyclav, Ceftazidime \\
\hline MRSA & Vancomycin, Linezolid, Tigecycline \\
\hline $\begin{array}{l}\text { Streptococcus } \\
\text { pneumoniae }\end{array}$ & Ampicillin, Cefotaxime, Ceftriaxone \\
\hline $\begin{array}{l}\text { Streptococcus } \\
\text { pyogenes }\end{array}$ & Amoxycillin, Ampicillin, Cefotaxime \\
\hline $\begin{array}{l}\text { Pseudomonas } \\
\text { Ceftazidime,Ciprofloxacin }\end{array}$ \\
\hline Klebsiella & Piperacillin, Amikacin, Ceftazidime \\
\hline Escherichia coli & Amikacin, Gentamycin, Ceftriaxone \\
\hline
\end{tabular}

\section{Antibiotic Therapy}

Initial antibiotics given were Ampicillin with Cloxacillin (100mg/kg/day, q 8 hrly). Appropriate antibiotic course was given according culture and sensitivity pattern, most cases recoverd with good response.

Among cases from which Staphylococcus aureus was isolated, one was MRSA, treated with intravenous Vancomycin (40mg/kg/day, q 6hrly) with good response. Those with Pseudomonas and Klebsiella positive cultures were treated with Piperacillin and Amikacin, E.coli positive cultures with Ampiclox and Amikacin. Pneumococcus and Streptococcus positive cultures with Ampicillin and Cloxacill.

Those cases in which cultures were sterile, course of Ampicillin with Cloxacillin were continued with adequate clinical response.

Intravenous antibiotic therapy was continued during hospital stay and discharged with advise of oral antibiotics, Amoxicillin with clavulanate to complete antibiotic course of 4 to 6 weeks.
Radiological findings at the time of Diagnosis

\begin{tabular}{|l|c|}
\hline CHEST XRAY FINDINGS & NO. OF CASES \\
\hline PLEURAL EFFUSION & $35(100 \%)$ \\
\hline PYOPNEUMOTHORAX & $2(5.71 \%)$ \\
\hline CONSOLIDATION & $29(82.85 \%)$ \\
\hline COLLAPSE & $16(45.71 \%)$ \\
\hline MEDIASTINAL SHIFT & $12(34.28 \%)$ \\
\hline
\end{tabular}

Out of 35 cases in our study, chest xray suggestive of effusion in all cases, pyopneumothorax in 2 cases $(57.14 \%)$, consolidation in 29 cases, collapse in 16 cases and mediastinal shift in 12 cases.

\section{Ultrasound Chest Findings}

9 cases had mild effusion, 15 had moderate and 11 had severe effusion. Out of 35 cases, 19 cases (54.28\%) had loculated effusions

\begin{tabular}{|l|c|}
\hline MODES OF TREATMENT & NO. OF CASES \\
\hline ICT + ANTIBIOTICS & 32 \\
\hline $\begin{array}{l}\text { ICT + ANTIBIOTICS + } \\
\text { DECORTICATION }\end{array}$ & 3 \\
\hline
\end{tabular}

All cases were treated with antibiotics and intercostal tube drainage. 3 cases $(8.57 \%)$ which did not improve with intercostal tube drainage and had loculations, had undergone Decortication. Poor response to intercostal tube drainage may be due to late presentation and delayed intercostal tube insertion.

\section{Outcome of cases treated with ICT Drainage}

Out of 32 cases treated with Intercostal tube drainage and antibiotics, 25 cases (78.12\%) improved well , 3cases $(9.37 \%)$ has expired, 2 cases left hospital against medical advice before completion of treatment course, 2cases which did not improve have been referred to higher centre ,at request, for further management.

\section{Outcome of Decortication}

3 cases out of 35 , which did not improve after a course of antibiotic therapy and intercostal tube drainage, with persistent loculated effusion and pleural thickening were treated by decortication. 
All 3 cases were improved after surgical intervention.

\section{Duration of ICT Drainage}

Most of the cases, $16(45.71 \%)$ required Intercostal tube drainage with ICT in situ for $7-15$ days, less than 1 week in 12 cases $(34.28 \%)$. Drainage was required for more than 15 days in only 7 cases ( 20 $\%)$. Mean duration of ICT drainage is 11 days.

\section{Length of Hospital Stay}

Most patients, 21 cases $(60 \%)$ required hospitalization for 14 to 30 days. Only 4 cases (11.42\%), stayed in hospital for less than 14 days of which one expired after 11 days of treatment and two were discharged againt medical advice. 10 cases $(28.57 \%)$ were hospitalized for more than 30 days. Mean length of hospital stay was 24.2 days.

\section{Mortality}

No. of cases expired during hospital stay were three with $8.57 \%$ mortality. In our study, out of 21 males, 2 cases expired $(9.52 \%)$ and out of 14 females, 1 case expired $(7.14 \%)$. Compared to females, 1 case, mortality was more in males, 2 cases(66.66\% of expired cases). Of the 3 cases expired in our study, 1 case is from each age group of $<3 \mathrm{yrs}, 3-5 \mathrm{yrs},>5 \mathrm{yrs}$. All the children who expired were malnourished, below 50th percentile. Of these 3 cases, 2 are below 3rd percentile. Out of 3 cases expired in our study, 1 child $(33.33 \%)$ is HIV infected, already on ART. Death was due to severity of infection and late presentation to hospital

\section{Summary}

Paediatric empyema thoracis at King George Hospital, 'Visakhapatnam, a prospective study on 35 patients.

The objective of the study was to analyse and evaluate the occurence of empyema cases among PICU admissions; age and sex wise distribution, side and sex preference, seasonal variations, clinical presentations and associated lesions, bacteriological agents in etiology of empyema cases, management, out come, morbidity and mortality patterns in relation to age, sex and co morbid conditions.

These were the observations made in our study.

1) The occurrence of empyema cases among our PICU admissions was $0.6 \%$.

2) The male children are more affected than the female children, comprising of $60 \%$.

3) The lowest incidence (5.71\%) of empyema cases were seen in below 1 year age group and as the age advanced the incidence of cases gradually increased and maximum incidence $(45.71 \%)$ of cases were noted in 35 years age group. Incidence decreased after 5 yrs of age $(20 \%)$.

4) Right side (57.14\%) was predominantly affected. In one case both Right and Left sides are involved.

5) The incidence of cases during winter season was $37.14 \%$.

6) Fever (100\%) and Breathlessness (100\%) were the most common symptoms present in all cases followed by cough $(88.57 \%)$. Chest pain was present in $39.66 \%$.

7) Pleural fluid was an exudate and cytology has shown polymorphonuclear predominance in all the cases.

8) Majority of cases were culture negative (51.42\%) for pleural aspirates. Among the bacterial isolates staphylococcus aureus was the major organism (17.14\%). While others like Pneumococcus (11.42\%), Pseudomonas (8.57\%), Klebsiella (5. $71 \%$ ), E.coli (2 . $85 \%)$ and Beta- haemolytic Streptococci $(2.85 \%)$ were frequently isolated from pleural aspirate.

9) On radiological examinations of chest, Chest $\mathrm{X}$-Ray shows associated lung lesions like consolidation in $82.85 \%$, collapse in 45.71\%. Mediastinal shift seen in $34.28 \%$. Pyopneumothorax seen in 2 cases. Ultrasound chest shows loculated collection in 54.28\%. Most cases (42.85\%) had moderate amount of effusion. 
10) The key to successful management lies in effective pleural evacuation and re-expansion of the lung.

11) Most cases 32 out of 35 were treated with a combination of intravenous antibiotics and chest tube drainage alone. Mean length of ICT drainage was 11 days.

12) In 3 cases, which were not improved after intercostal drainage and antibiotics, probably due to late presentation to hospital and delayed insertion of intercostal tube, HRCT chest revealed organized empyema and underwent Decortication in Dept of cardio thoracic surgery KGH. In all the cases which underwent surgery, recovery is uneventful. VATS is not available at KGH. Fibrinolytics were not used in our study due to cost restraint.

13) 2 cases who were not improved with chest tube drainage and antibiotics and shown severe pleural thickening in HRCT Chest, were referred to higher centre at the request of their parents.

14) 2 cases were discharged against medical advice with completion of adequate course of treatment.

15) Mean length of hospital stay was 24.2 days.

16) 3 cases of empyema on ICT drainage expired. Mortality of the illness was $8.57 \% .1$ case died of respiratory failure due to late presentation, 2 cases died due to associated septicemia.

17) Most of the deaths are seen among male children.

18) Mortality in all the three age groups ( $<3 y r s$, 3-5 yrs, $>5$ yrs) were equal, one in each.

19) Malnutrition was present in $100 \%$ of cases in the study group. Out of 3 cases expired, 2 cases have weight $<3$ rd percentile, 1 cases is between 3rd- 50th percentile.

Out of 2 HIV infected cases one case died due to severe infection with septicemia and late presentation to hospital, the other case recovered uneventfully. Proportion of deaths in HIV infected cases among total empyema deaths was $33.33 \%$.

\section{Conclusions}

- The occurence of Empyema thoracis among PICU admissions during the study period is $0.6 \%$.

- The occurence of Empyema thoracis is more in male children.

- The occurrence of empyema is less below the age of 1 year and as the age advances the incidence is gradually increasing and maximum incidence of cases seen between 3-5 $\mathrm{y}$.

- The occurrence of Empyema is more in male children, below the age of 1 year.

- The occurrence of Empyema is more common in malnourished children.

- All the HIV cases (2 cases) presented similar to normal children and the outcome is good in 1 case, one case died due to severe infection with septicemia and late presentation.

- High incidence of Empyema is seen in winter season (Nov. to Feb.)

- The most frequent presenting symptoms of Empyema in all cases are Fever and Breathlessness followed by cough, less frequently by chest pain

- Right sided Empyema is more common.

- Empyema rarely occur bilaterally.

- Blood culture was negative in most of the cases.

- Pleural fluid culture was negative in majority of cases $(51.42 \%)$.

- Staphylococcus aureus is the commonest organism causing Empyems thoracis.

- The incidence of Staphylococcal Empyema is more common below 3 years age.

- Chest X-ray shows majority of empyema cases to be associated with underlying parenchymal disease like consolidation.

- Ultrasound chest shows majority to have moderate amount of effusion at diagnosis and most collections are loculated. 
- The standard treatment for Empyema thoracis is by a combination of intravenous antibiotics and chest tube drainage, in some patients decortications is required, probably due to late presentation to hospital and delayed insertion of intercostal tube

- The morality rate of Empyema is $8.57 \%$. Higher mortality rate is seen in male sex.

- Early diagnosis, prompt and effective treatment of respiratory infections particularly Pneumonias will lower the incidence of Paediatric Empyema thoracis.

- Similar outcomes are seen in HIV cases also with early diagnosis and treatment

- Early diagnosis, early referral and prompt treatment with antibiotics and ICT drainage reduce the mortality associated with empyema thoracis.

\section{References}

1. Nelson text book of pediatrics $20 \mathrm{t} \mathrm{h}$ edition 2015, p 2131 - 2135.

2. Abstract from 00ty pedicon 2005 journal study by suresh et.al at ICH Chennai paediatric on call

3. J Indian ass of paediatric surgeons 2010 jan-mar 15(1)9-14. Study by Prema menon of PGIMER et.al.

4. Internet journal of surgery 2008 vol14 num1. Modified Feb 2009 14;19;20-0600. A Study by Ashish Gupta et.al of MGM Med coll Indore.

5. Professional medical journal sep 2010;17(3) p 464-471. Study by Muhammad Saleem et.al of ICH Lahore.

6. South African journal of child health Oct 2007 vol3 p 121-126. Study by Zampoli et al Cape town,

7. J. Archives of diseses in child health 2003 Nov 88(11) p 1009-1014. 10 y retrospective study by Baranwal et.al from PGIMER.

8. WHO Bulleiten by Stephen et.al vol 86;2008num 5 p 328-416

9. British journal of surgery Dec2005vol79 p531-534. Study by Desai et.al Zambia

10. IAP J 2002 39; P 145-157. Study by Meenu Singh et.al at PGIMER.

11. OP Ghai essential pediatrics 8th edition, $p$ 395.

12. IAP Text book of pediatrics 5 th edition, $p$ $478-480$.

13. VATS procedure. Post graduate med j2000 76; p 547-550. 